\title{
Sub kGy photon irradiation alterations in graphite
}

\author{
D.A. Bradley ${ }^{a, *}$, Siti Nurasiah Mat Nawi ${ }^{\text {a }}$, Mayeen Uddin Khandaker ${ }^{a}$, K.S. Almugren ${ }^{\text {b }}$, S. \\ F Abdul Sani ${ }^{\mathrm{c}}$
}

${ }^{a}$ Centre for Biomedical Physics, School of Healthcare and Medical Sciences, Sunway University, 47500, Bandar Sunway, Selangor, Malaysia

${ }^{\mathrm{b}}$ Department of Physics, Princess Nourah Bint Abdulrahman University, Riyadh, Saudi Arabia

${ }^{\mathrm{c}}$ Department of Physics, University of Malaya, 50603, Kuala Lumpur, Malaysia

\section{A R T I C L E I N F O}

\section{Keywords:}

Luminescence

Polymer pencil-lead graphite

Trapping parameter

Photoluminescence spectroscopy

$\mathrm{X}$-ray diffraction

\begin{abstract}
A B S T R A C T
Present work concerns polymer pencil-lead graphite (PPLG) and the potential use of these in elucidating irradiation-driven structural alterations. The study provides detailed analysis of radiation-induced structural interaction changes and the associated luminescence that originates from the energy absorption. Thermally stimulated emission from the different occupied defect energy levels reflects the received radiation dose, different for the different diameter PPLGs. The PPLG samples have been exposed to photon irradiation, specifically $\mathrm{x}$-ray doses ranging from 1 to $10 \mathrm{~Gy}$, extended to $30-200 \mathrm{~Gy}$ through use of a ${ }^{60} \mathrm{Co}$ gamma-ray source. Trapping parameters such as order of kinetics, activation energy and frequency factor are estimated using Chen's peak-shape method for a fixed-dose of $30 \mathrm{~Gy}$. X-ray diffractometry was used to characterize the crystal structure of the PPLG, the aim being to identify the degree of structural order, atomic spacing and lattice constants of the various irradiated PPLG samples. The mean atomic spacing and degree of structural order for the different diameter PPLG are found to be $0.3332 \mathrm{~nm}$ and $26.6^{\circ}$ respectively. Photoluminescence spectra from PPLG arising from diode laser excitation at $532 \mathrm{~nm}$ consist of two adjacent peaks, $602 \mathrm{~nm}$ (absorption) and $1074 \mathrm{~nm}$ (emission), with mean energy band gap values within the range $1.113-1.133 \mathrm{eV}$.
\end{abstract}

\section{Introduction}

Over the past 90 years and more the various effects of ionizing radiation have been well documented, typically in regard to deterministic and stochastic biological risks and mostly at sub-Gy levels, notably in as much as these concerns reach the public eye. From as long ago as 50 years, irradiations of mammalian tissues have been associated with DNA alterations, not least cancer and genetic mutation stochastics (Hall and Giaccia, 2006). Less well publicized have been the very large efforts, daily carried out, on the development of high-strength highly resilient composite materials via in particular irradiation-promoted cross-linking at tens through to hundreds of kGy, delivered using gamma-ray and accelerated electron sources. With benefits versus risk now being well appreciated and under highly controlled conditions, a wide-range of safe technology applications have evolved, not just in medicine but also in industry, nuclear energy and radiation source utilization included. In regard to the study of structural integrity, major efforts have been made towards the development of lighter materials of greater strength, carbon fibre technology being one such area of thrust, including the carbon fibres used in radiotherapy suite treatment couches, aircraft wings etc. In regard to graphitic materials, the focus of current interest, much less studied have been the possible post-production signs of relatively low dose (sub kGy) irradiation-promoted alterations in the materials. This differentiates present work from the extensive studies that have been carried out on MGy neutron dose material degradations in the graphite moderators of nuclear reactors, impacting on performance and the life-cycle of these media. However, thus said, for dose effects on carbon fibres see for instance Sharp and Burnay (1974).

Key to such irradiated material studies, whether they be in regard to low or high dose, is the ability to evaluate radiation dose, dosimeters and dose simulations playing key roles in radiation protection monitoring and quality control. In present work using external beam x-ray facilities of note is that the doses from these are known through use of ionisation chambers. An acknowledged aside is a potential for dosimetric material performance alteration in long-term use of the graphite-based ion chambers, commonly applied as noted in control of irradiation dose delivery.

With these opening statements, present work concerns the structural

\footnotetext{
* Corresponding author.

E-mail address: d.a.bradley@surrey.ac.uk (D.A. Bradley).
} 